\title{
EVALUACIÓN EXPERIMENTAL DE Ulomoides dermestoides (TENEBRIONIDAE) COMO HOSPEDERO INTERMEDIARIO DE Hymenolepis diminuta
}

\author{
Diana Villegas-Fudino ${ }^{1, a}$, Manuel Tantaleán-Vidaurre ${ }^{2, b}$
}

\begin{abstract}
RESUMEN
El objetivo del estudio fue determinar si Ulomoides dermestoides se comporta como hospedero intermediario de Hymenolepis diminuta, por lo que cien de ellos se infectaron experimentalmente con huevos del cestodo. Como resultado se obtuvo que en el $92 \%$ de los insectos se formaron cisticercoides que, inoculados en ratas de laboratorio, desarrollaron el estadio adulto con cuyos huevos se repitieron nuevas infecciones obteniendo los mismos resultados, por lo que se puede señalar que $U$. dermestoides experimentalmente se comporta como hospedero intermediario.
\end{abstract}

Palabras clave: Hymenolepis diminuta; Ulomoides dermestoides; Estudios experimentales (fuente: DeCS BIREME).

\section{EXPERIMENTAL EVALUATION OF Ulomoides dermestoides (TENEBRIONIDAE) AS AN INTERMEDIATE HOST OF Hymenolepis diminuta}

\begin{abstract}
The aim of the study was to determine if Ulomoides dermestoides behaves as an intermediate host of Hymenolepis diminuta, therefore one hundred of them were experimentally infected with tapeworm eggs. As a result, it was found that in $92 \%$ of the insects formed cysticercoids which, inoculated in laboratory rats, developed the adult stage. These adults produced eggs with which the infection process was repeated, obtaining the same results. Therefore it can be said that $U$. dermestoides experimentally acts as an intermediate host.
\end{abstract}

Key words: Hymenolepis diminuta; Ulomoides dermestoides; Experimental studies (source: MeSH NLM).

\section{INTRODUCCIÓN}

El insecto Ulomoides dermestoides (= Palembus d.), o "escarabajo del maní", es un coleóptero de la familia Tenebrionidae al que se le conoce como plaga de granos almacenados ${ }^{(1)}$. Se le encuentra en hábitats terrestres, asociados con ambientes secos, debajo de la madera en descomposición ${ }^{(2)}$. Se reproduce adecuadamente en los granos de maní, maíz, arroz, sorgo, frijol mungo y soya. Según referencia popular, en la China y Malasia, las heces de este insecto curan la indigestión, las afecciones cardiacas, mialgias, enfermedades renales y el asma ${ }^{(3,4)}$

La medicina tradicional practicada en varios países latinoamericanos, incluyendo el Perú, promociona la ingestión de escarabajos como $U$. dermestoides con fines terapéuticos para tratar síntomas de una amplia gama de enfermedades como asma, artritis, cáncer, diabetes, enfermedad de Parkinson, impotencia sexual, problemas oculares, psoriasis, quistes ováricos, entre otros; y aunque no existen referencias sobre el seguimiento clínico y/o de experimentación científica en los pacientes que practican la coleopteroterapia, su uso y eficacia se divulga a partir de testimonios ${ }^{(5)}$. Sin embargo, la población que los consume podría estar en riesgo de ingerir formas infectantes de parásitos que utilizan artrópodos como vectores; por esta razón, en el presente trabajo, se investiga si $U$. dermestoides puede ser un importante hospedero intermediario de Hymenolepis diminuta.

\section{EL ESTUDIO}

Estudio de tipo experimental que se llevó a cabo en el Laboratorio de Parasitología de la Facultad de Veterinaria y Zootecnia de la Universidad Peruana Cayetano Heredia en Lima, Perú, durante los meses de septiembre

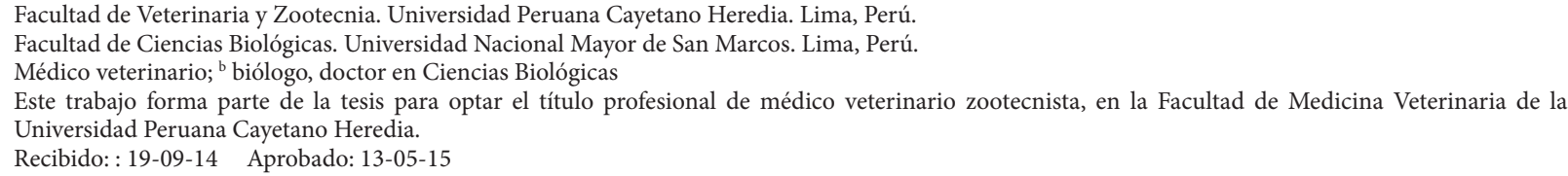

Citar como: Villegas-Fudino D, Tantaleán-Vidaurre M. Evaluación experimental de Ulomoides dermestoides (Tenebrionidae) como hospedero intermediario de Hymenolepis diminuta. Rev Peru Med Exp Salud Publica. 2015;32(3):515-8. 
a diciembre de 2012. La investigación contó con la aprobación del Comité Institucional de Ética para uso de animales, de la Universidad Peruana Cayetano Heredia.

\section{MUESTRAS}

Para el estudio, se emplearon cien especímenes de Ulomoides dermestoides, cien de Tribolium confusum, que corresponden a nuevas generaciones de un criadero, por lo tanto, libres de infección y 1 rata infectada con Hymenolepis diminuta, proporcionados por el Laboratorio de Parasitología en Fauna Silvestre y Zoonosis, de la Facultad de Ciencias Biológicas de la Universidad Nacional Mayor de San Marcos. La infección de ambos insectos se realizó de acuerdo con la metodología descrita por Voge y Heyneman ${ }^{(6)}$.

\section{PROCESAMIENTO DE MUESTRAS}

La rata infectada se sacrificó con sobredosis de cloroformo. Los cestodos recuperados se lavaron en solución salina y se separaron los proglótidos grávidos para la obtención de los huevos. Previamente, los coleópteros, en ayunas durante 10 días, se colocaron en placas de Petri que contenían hojuelas de avena con una suspensión de huevos del parásito o pequeños segmentos de proglótidos grávidos. Cada 24 h se sacrificaron entre 5 y 10 insectos de cada especie, con la finalidad de observar la evolución de las oncósferas hasta el desarrollo completo del cisticercoide. Los cisticercoides obtenidos se inocularon en cinco ratas jóvenes de la cepa Swiss Webster, a razón de 1-20 cisticercoides por animal. A partir del día 10 se analizaron las heces hasta observar los huevos del parásito. Finalmente, se sacrificaron las ratas y se diseccionó el intestino delgado para recuperar los cestodos adultos; de algunos de ellos se separaron proglótidos grávidos para obtener huevos, mientras que los cestodos restantes se fijaron con formol al $10 \%$. Con los huevos obtenidos de estas infecciones se volvieron a infectar nuevos insectos y ratas hasta el desarrollo final del cestodo.

Todos los estadios larvarios de la infección de $U$. dermestoides se fotografiaron en fresco y sin coloración en un microscopio Leica con cámara digital incorporada, agregando a cada imagen la escala métrica respectiva. Los proglótidos maduros del parásito adulto se colorearon con carmín acético de Semichon.

\section{HALLAZGOS}

El porcentaje de infección en $U$. dermestoides con las diferentes etapas evolutivas del cisticercoide fue del $92 \%$. A partir del octavo día se obtuvieron entre 10 y 94 cisticercoides por insecto. La descripción de los siete estadios evolutivos del cisticercoide de $H$. diminuta en $U$. dermestoides se presentan en la Figura 1. En la Tabla 1 se comparan los resultados obtenidos en el presente estudio con los anteriormente publicados ${ }^{(6)}$.
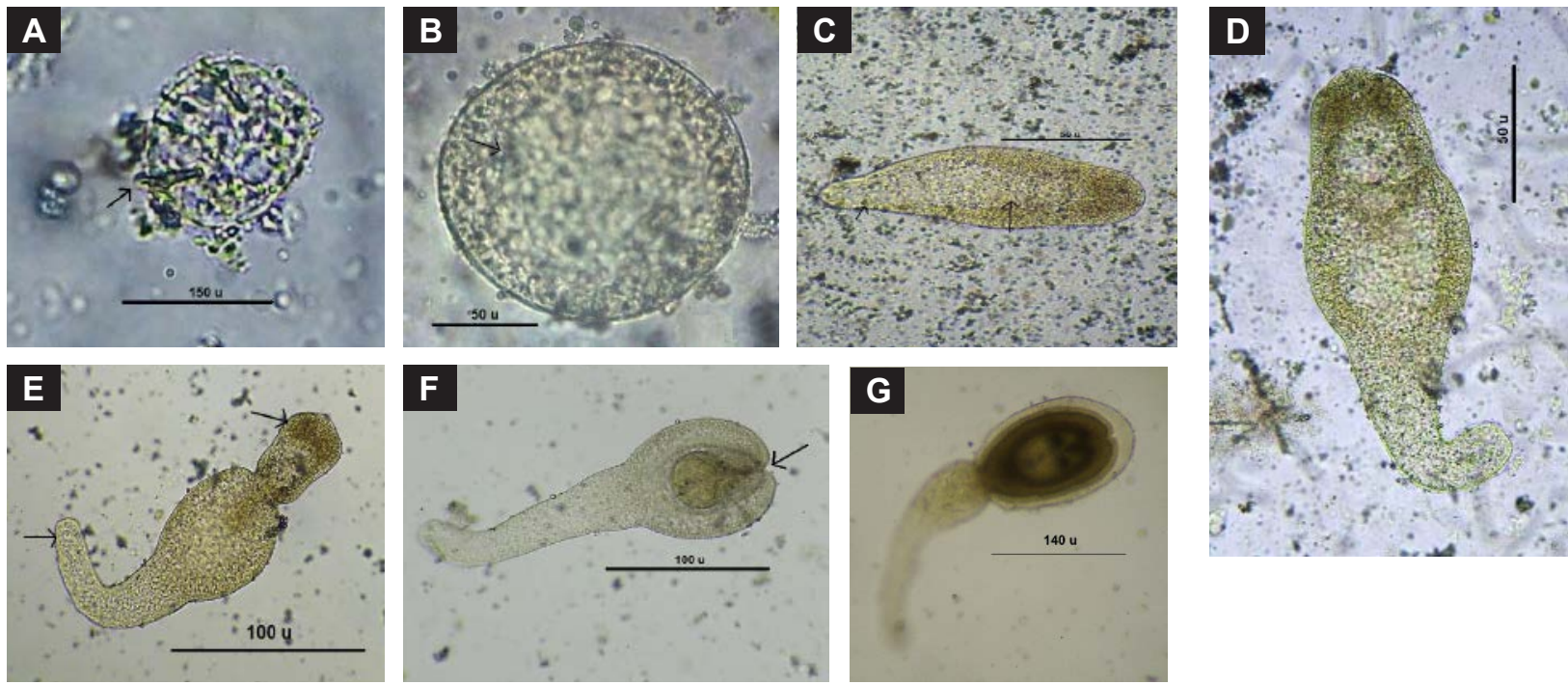

Figura 1. Estadios evolutivos del cisticercoide de H. diminuta en la U. dermestoide A). A las $24 \mathrm{~h}$. La oncósfera tiene forma esférica y los ganchos se van situando periféricamente sin perder su característica morfológica. B). Dos a tres días. Adquiere mayor tamaño y se va formando una cavidad en la parte media. Los ganchos se van perdiendo y pueden observarse en un extremo o alrededor de la esfera. C). Tres a cuatro días. Se aprecia alargamiento del cuerpo y la cavidad interna se hace más notoria. Los ganchos son más pequeños y van desapareciendo. D). Cuatro a cinco días. Continúa alargándose y se va formado un estrechamiento anterior y otro posterior más delgado. E). Seis a siete días. Las divisiones corporales están bien definidas; en el extremo anterior se aprecia la formación del escólex rudimentario y en el extremo opuesto, el apéndice caudal. En algunas larvas se observan restos de ganchos, generalmente en la cola. F). Siete a ocho días. El escólex se encuentra retraído en la cavidad del cuerpo y el apéndice caudal está más diferenciado. G). Ocho a nueve días. El cisticercoide se encuentra completamente formado, mostrando el escólex invaginado y rodeado de membranas, el extremo posterior con un apéndice caudal en la que se observan algunos ganchos degenerados 
Tabla 1. Comparación de la evolución del cisticercoide según Voge y Heyneman (1957)

\begin{tabular}{cclll}
\hline & & \multicolumn{1}{c}{ Voge y Heyneman (1957) } & \multicolumn{1}{c}{ Presente trabajo } \\
\hline Estadio & Días & Principales características & Días & Principales características \\
1 & $0-2$ & Esférico, sin cavidad & $1-1,5$ & Esférico, con ganchos \\
2 & $2-3$ & Esferoidal, cono cavidad excéntrica & $2-3$ & Formación de cavidad central \\
3 & $3-5$ & Piriforme. Cuerpo con dos divisiones & $3-4$ & Alargamiento del cuerpo. Cavidad notoria \\
4 & $5-6$ & División pronunciada. Retracción del escólex & $4-5$ & Mayor tamaño. aparece división del cuerpo \\
5 & $6-8$ & Aumento de la porción media del cuerpo. & $6-7$ & División definida del cuerpo. Formación del escólex \\
6 & & Madurez & $7-8$ & Retracción del escólex y apéndice caudal. \\
\hline & & & $8-9$ & Cisticercoide maduro \\
\hline
\end{tabular}

Tabla 2. Desarrollo del estadio adulto de $H$. diminuta en ratas

\begin{tabular}{ccccc}
\hline \multirow{2}{*}{ Ratas } & \multirow{2}{*}{$\begin{array}{c}\text { Cisticercoides } \\
\text { inoculados }\end{array}$} & \multicolumn{3}{c}{ Presencia de huevos en heces } \\
\cline { 3 - 5 } & 22 & 16 días & 17 días & 20 días \\
\hline 1 & 17 & + & + & + \\
2 & 20 & + & + & + \\
3 & 15 & - & + & + \\
4 & & - & + & + \\
\hline
\end{tabular}

La Tabla 2 muestra el proceso de desarrollo del adulto en la rata, hasta la observación de huevos en las heces. La vista de los proglótidos nos permitió observar que, morfológicamente, las gónadas y otras estructuras genitales tenían características normales; además, los huevos obtenidos de los proglótidos grávidos nuevamente formaron cisticercoides morfológicamente normales en $U$. dermestoides.

\section{DISCUSIÓN}

$H$. diminuta es un cestodo cosmopolita que requiere de un hospedero intermediario para completar su ciclo evolutivo. El hospedero definitivo lo constituyen varias especies de roedores, especialmente Rattus spp. y el intermediario son diversos insectos ${ }^{(7)}$, pero el más importante como vehículo de infección en el hombre es Tribolium spp.

En el Perú, la hymenolepiosis producida por $H$. diminuta es una zoonosis poco difundida; sin embargo, se ha comunicado que en algunas lugares de interior del país la infección humana es alta como ocurre en las localidades de Cajamarca y Tingo María ${ }^{(8,9)}$.

En trabajos previos ${ }^{(6,10)}$ se ha estudiado el desarrollo del cisticercoide de $H$. diminuta y del adulto durante los primeros 5 días en los hospederos naturales, T. confusum y rata respectivamente; y aunque se establecieron arbitrariamente cinco estadios evolutivos para el cisticercoide, ambos modelos se han tomado como patrones para la presente investigación. Nosotros, de acuerdo con nuestras observaciones, también hemos establecido arbitrariamente siete etapas en el desarrollo del cisticercoide, lo cual nos permite diferenciar mejor las etapas del desarrollo.
Si comparamos los resultados obtenidos en el presente estudio con los de Voge ${ }^{(6,11)}$, observamos que el desarrollo del cisticercoide se encuentra dentro de los parámetros normales tanto en los tiempos de evolución como en las características morfológicas; así, en el presente trabajo observamos que el $92 \%$ de $U$. dermestoides se infectaron y desarrollaron el metacestodo hasta la última fase empleando entre 8 y 10 días, semejante a los 6-8 días en $T$. confusum ${ }^{(6)}$; la diferencia de 2 días se debería a la especie de artrópodo utilizada y a las condiciones ambientales del experimento. En el presente trabajo, el insecto infectado se mantuvo a temperatura ambiente. Del mismo modo, el desarrollo de los diferentes estadios, desde oncósfera hasta cisticercoide, es semejante, como por ejemplo, de 3 a 6 días para la formación del estadio 3 (alargamiento del cuerpo y presencia de cavidad en la parte central) y de un máximo de 9 días para el desarrollo completo del cisticercoide.

Con relación al desarrollo del estadio adulto de $H$. diminuta, se observó que este se manifestó por el hallazgo de huevos en las heces entre los 16 y 20 días de la inoculación con cisticercoides, lo que significa que los cisticercoides procedentes de Ulomoides no están afectados en su capacidad infectiva, aun cuando la cantidad de cisticercoides inoculados fue variable. Por otro lado, los estadios adultos recuperados fueron morfológicamente normales y mostraron la vesícula seminal con espermatozoides y el útero con huevos en diferentes estadios de formación. No se observó ningún proglótido con alteraciones morfoanatómicas, aun en la segunda generación.

La longitud de especímenes adultos recuperados de las ratas fue de 45 a $60 \mathrm{~cm}$, por lo tanto, no se apreció disminución notable aun cuando se inocularon varios cisticercoides, coincidiendo con Stradowski ${ }^{(12)}$. El estadio adulto afecta a las ratas porque les causa atrofia de las vellosidades intestinales por compresión, degeneración y descamación de las células epiteliales y excesiva producción de mucina en la mucosa intestinal y lúmina; ocasionalmente se ha observado infiltración eosinofílica ${ }^{(13)}$.

Todos los hallazgos obtenidos en este trabajo nos indican que $H$. diminuta se desarrolla, experimentalmente, en forma normal en las fases de cisticercoide y adulto 
cuando el hospedero intermediario es $U$. dermestoides. Por los resultados obtenidos en el presente estudio, se puede afirmar que $U$. dermestoides se comporta como un hospedero intermediario altamente susceptible a $H$. diminuta y que por su tamaño, puede albergar mayor número de cisticercoides. Esta última observación tendría una aplicación práctica porque puede emplearse como una forma de obtener un buen número de cisticercoides que podrían ser utilizados en estudios biológicos ${ }^{(14)}$. Se ha demostrado, experimentalmente, que $U$. dermestoides también se comporta como hospedero intermediario de Macracantorhynchus hirudinaceus (3), un acantocéfalo intestinal común en cerdos y raro en el hombre ${ }^{(15,16)}$.

Se debe señalar que las especies de $U$. dermestoides que se crían con fines terapéuticos, corren el riesgo de infectarse con huevos de helmintos parásitos, en este caso de $H$. diminuta, por la presencia de ratas y cucarachas que son atraídas por el alimento, por lo que se sugiere protegerlos adecuadamente del contacto con heces de animales como las ratas, para evitar que actúen como hospederos intermediarios y transmitan parásitos que afecten al hombre.
Este estudio no tuvo limitación en su ejecución por cuanto los insectos estudiados y las ratas se crían fácilmente en cualquier laboratorio.

En conclusión, se demuestra experimentalmente que $U$. dermestoides es altamente susceptible a la infección por $H$. diminuta, por lo que se le puede considerar un hospedero intermediario; por ello es importante proteger adecuadamente los criaderos de estos insectos para evitar el contacto con ratas que puedan contaminar el alimento con huevos del cestodo y luego infectar al hombre que los ingiere vivos para el tratamientos de varias enfermedades en diferentes países.

Contribuciones de autoría: DVF participó en la concepción y diseño del estudio, recolección del material y obtención de resultados, búsqueda bibliográfica, redacción del artículo y aprobación de la versión final. MTV participó en el análisis e interpretación de datos, revisión del artículo y aprobación de la versión final.

Financiamiento: autofinanciado.

Conflictos de interés: los autores declaran no tener conflictos de interés.

\section{REFERENCIAS BIBLIOGRÁFICAS}

1. Gross L, Galaschi J, Corseuil E. Imobilização de larvas de Ulomoides dermestoides (Coleoptera, Tenebrionidae) sob baixa temperatura. Biociências. 2005;13(2):119-21.

2. Chacón R, Villalba V, Moreira I. Descripción anatómica de los órganos genitales y glándulas secretoras abdominales del escarabajo Ulomoides dermestoides (fairmare 1893) (Coleoptera:Tenebrionidae). Tecnología en Marcha. 2009;22(4):4565.

3. Kleemann I, Luz E, Tonin V. Experimental Infection of Palembus dermestoides by Macracanthorhynchus hirudinaceus larvae. Acta Biol Par Curitiba. 1990;19(1-4):45-9.

4. Tobón F, Gutiérrez G, Mejía M. Evaluación del perfil neurofarmacológico del aceite de Ulomoides dermestoides (Coleoptera: Tenebrionidae). Rev Colomb Entomol. 2011;37(2):251-5.

5. German F. Sobre el uso de Ulomoides dermestoides (Chevrolat, 1878), (Coleoptera, Tenebrionidae, Diaperini) en la coleopteroterapia: Informe de un caso en Ixtapa, Jalisco, México. Boln Asoc Esp Ent. 2010;34(3-4):419-422.
6. Voge M, Heyneman D. Development of Hymenolepis nana and Hymenolepis diminuta (Cestoda: Hymenolepidae) in the intermediate host Tribolium confusum. California: University of California; 1957.

7. Heicher DS, Gallati W. Three new hosts for the cysticercoid of Hymenolepis diminuta. Ohio J Sci. 1978;78(3):14951.

8. Náquira C, Delgado E, Tantaleán M, Náquira F, Elliot A. Prevalencia de enteroparásitos en escolares de los distritos de San Juan y Magdalena (Departamento de Cajamarca) 1971. Rev Per Med Trop. 1973;2(1):37-41.

9. Huiza A, Tantaleán M, Juárez $D$. Observaciones sobre la presencia de cuatro enteroparásitos en la región de Rupa-Rupa en el Dpto. de Huánuco. Bol Med Trop Lima. 1986;5(1):7-9.

10. Goodchild CG, Harrison DL. The growth of the rat tapeworm, Hymenolepis diminuta, during the first five days in the final host. J Parasitol. 1961;47:819-29.

11. Voge M. Studies in Cysticercoid Histology. I. Observations on the fully developed cysticercoid oh Hymenolepis diminuta (Cestoda: Cyclophyllidea). P Helm Soc Wash.1960;27(1):32-6.
12. Stradowski M. The development of Hymenolepis diminuta tapeworms of inbred line WMS iL1 in rats of the WAG alb. race in primary and secondary infections of varying intensity. Helminthologia. 2004;41(1):9-14.

13. Goswami R, Mohan S, Kataria M, Somvanshi R. Clinicopathological studies on spontaneous Hymenolepis diminuta infection in wild and laboratory rats. Braz J Vet Pathol. 201 1;4(2):103-11.

14. Lethbridge RC. 1971. The locust as an intermediate host for Hymenolepis diminuta. J Parasitol. 1971;57(2):445-6.

15. Tesana S, Mitrchai J, Chunsuttwat S. Acute abdominal pain due to Macracanthorbynchus birudinaceus infection: A case report. Se Asian J Trop Med. 1982;13(2):262-4.

16. Neafie RC, Marty AM. Unusual infections in humans. Clin Microbiol Rev. 1993;6(1):34-56.

Correspondencia: Diana Villegas-Fudino Dirección: Calle Diego Quispe Tito 267 Urb. Pando 7.a Etapa, San Miguel. Lima, Perú Teléfono: (+511) 5660453

Correo electrónico: dianavf314@gmail.com 